\title{
Relación entre las unidades de vegetación y la microtopografía en un pastizal ubicado en un sector mal drenado de Argentina
}

\author{
Beatriz Martín', Oscar Sosa ${ }^{2}$, Sergio Montico ${ }^{2}$ y Gabriel Zerpa ${ }^{2}$ \\ ${ }^{1}$ Especialidad Forrajes, ${ }^{2}$ Especialidad Manejo de Tierras. Facultad de Ciencias Agrarias. \\ Universidad Nacional de Rosario. CC N 14 (S 2125 ZAA), Zavalla, Santa Fe, Argentina.
}

\begin{abstract}
B. Martín, O. Sosa, S. Montico, and G. Zerpa. 2007. Relationship between vegetation units and microtopography of a pasture located in a poorly drained sector of Argentina. Cien. Inv. Agr. 34(2):103-113. The spatial distribution of vegetable communities in depressed landscapes from Rolling Pampa (Pampa Ondulada), Argentina is frequently heterogeneous. The objective of this work was to study a possible relationship between topography and functional and structural characteristics of vegetation in a pasture located in a depressed and poorly drained area. The study was carried out in a sector next to a dell from the flowing Ludueña basin. The course of Ludueña extends between $32^{\circ} 46^{\prime}$ and $33^{\circ} 07^{\prime} \mathrm{S}$ and $60^{\circ} 39^{\prime}$ and $61^{\circ} 07^{\prime} \mathrm{W}$ in the Santa Fe province of Argentina. The identification of the plant communities and a topographical study allowed us to recognize three highly productive forage units. In each unit, primary production, abundance and cover were determined. Plant units were established accordingly. The results obtained suggest that vegetation cover and vegetation abundance from depressed landscapes are highly related with microtopography.
\end{abstract}

Key words: Communities, forage units, depressed landscapes. microtopography, vegetable.

\section{Introducción}

La Pampa Ondulada es una de las subregiones de la planicie pampeana argentina. Abarca una angosta franja al occidente de los ríos Paraná y de la Plata, que va desde el río Carcarañá en Santa Fe (al norte) hasta las inmediaciones de Punta Indioen Buenos Aires; mientras que hacia el oeste y el suroeste los límites son indefinidos. Debe su nombre al relieve predominantemente ondulado que la caracteriza. Presenta lomadas suaves, con gradientes de pendiente inferiores al $2 \%$, aunque hay partes que superan el $3 \%$. Los suelos se han formado a partir de sedimentos loéssicos de granulometría fina (INTA, 1983). Esta subregión es, en general, la mejor drenada de la gran llanura; sin embargo, presenta sectores deprimidos, casi siempre próximos a cursos de agua (INTA, 1983). Allí, las limitaciones de drenaje derivan

Recibido 12 de noviembre 2006. Aceptado 02 de abril 2007.

12 Dirigir correspondencia a B. Martín: bmartin@argentina.com de la ubicación en el paisaje y de la escasa profundidad de la napa freática (Mosconi et al., 1981). Estos ambientes abarcan un variado mosaico de suelos y se caracterizan por una conjunción de problemas de productividad (mal drenaje, anegamiento, salinidad y alcalinidad), determinante, en numerosos casos, de una baja aptitud agrícola. Por ello es que suelen destinarse a la producción pecuaria, en base a pasturas naturales o mejoradas.

En cualquier condición ambiental la dinámica de la vegetación pastoril es de alta complejidad y está influenciada por la interacción de aspectos tales como la historia y la presión de pastoreo, las precipitaciones y las propiedades del suelo (Milchunas y Lauenroth, 1989). La dificultad para llevar a cabo un uso sostenible de estos pastizales aumenta en aquellos que poseen menor productividad primaria (Behnke Jr. et al., 2000).

Las características topográficas y de drenaje propias de las tierras bajas determinan un 
comportamiento particular en los flujos materiales y energéticos. En esa dinámica adquieren importancia los microrrelieves y dominan las relaciones geoquímicas bidireccionales (González Bernaldez, 1981). Las diferencias en las intensidades de las relaciones geoquímicas existentes entre microrrelieves dan lugar a una diversidad de microambientes. Precisamente, en los paisajes deprimidos se presenta alta variabilidad espacial de propiedades químicas, físicas y biológicas del suelo. Esto ocurre en distancias relativamente cortas, circunstancia que, en muchos casos, permite la existencia de un abigarrado mosaico de suelos con características disímiles (Allen y Mc Intosh, 1997; Corwin et al., 2003).

La heterogeneidad espacial de ciertos componentes de los paisajes deprimidos destinados a pasturas, como son el drenaje y las propiedades edáficas, interactúa en forma compleja con el clima y el pastoreo para determinar la distribución de la vegetación en el terreno (Sala et al., 1986; Chaneton y Lavado, 1996; Onkware, 2000; Corwin et al., 2003).

El conocimiento de la estructura y del funcionamiento de un pastizal es requisito previo a la planificación de su uso. Las características florísticas estructurales del tapiz (Braun-Blanquet, 1979) condicionarán la preferencia y los hábitos de consumo de los herbívoros (Deregibus et al., 2001).

Algunos de los factores modeladores de la estructura de la vegetación a escala de predio son la topografía, tipo de suelo y condiciones micro-climáticas (Hartnett y Fay, 1998), la profundidad de la napa de agua y su movimiento dentro del perfil del suelo (Batista y León, 1992) y la interacción entre ellos (Pastor et al., 1987; Coffin y Lauenroth, 1988; Pastor y Post, 1993; Archer et al., 1995).

Diferentes autores señalan que en condiciones climáticas con poca variación y a un nivel regional, los factores edáficos y microtopográficos determinan las diversas formaciones vegetales a una escala local (Stephenson, 1990; Goslee et al., 1997; Sánchez-Sánches e Islebe, 2002).
En ambientes deprimidos la microtopografía modelaría la estructura de la vegetación. El conocimiento de esa relación puede ser útil para evaluar la funcionalidad del pastizal. Se sostiene la hipótesis de que la distribución aérea de los atributos topográficos influye en un alto porcentaje en la variabilidad espacial de las unidades forrajeras. Por lo tanto, el objetivo de este trabajo fue estudiar la relación entre las características estructurales y funcionales de la vegetación con la topografía, en un pastizal ubicado en un área deprimida y mal drenada.

\section{Materiales y métodos}

\section{Area de estudio}

El estudio se realizó en el Campo Experimental de la Facultad de Ciencias Agrarias (Universidad Nacional de Rosario), ubicado en las proximidades de la localidad de Zavalla, provincia de Santa Fe, Argentina, a los $33^{\circ}$ de latitud $\mathrm{S}$ y a los $67^{\circ}$ de longitud $\mathrm{O}$. El clima del área es húmedo-subhúmedo mesotermal, con un promedio anual de precipitaciones de 987 $\mathrm{mm}$ y una temperatura media anual de $17^{\circ} \mathrm{C}$ (datos de la Estación Meteorológica Zavalla, Servicio Meteorológico Nacional, Argentina; promedios de los 20 años anteriores al período experimental). Durante el verano existen numerosas jornadas con temperaturas máximas absolutas superiores a $30^{\circ} \mathrm{C}$; por otra parte, entre el 70 y el $75 \%$ de las lluvias ocurren en otoño y primavera, mientras que de diciembre a febrero el balance hídrico medio presenta las mayores deficiencias.

El lugar de trabajo se encuentra al pie de la pendiente lateral que se extiende hacia una cubeta elongada. A través de esta última discurre un canal tributario de la cuenca del arroyo Ludueña. El sector tiene una superficie de 74 ha y se caracteriza por un mal drenaje, con un pastizal natural bajo uso exclusivo ganadero.

En este lugar se relevaron las comunidades vegetales existentes a principios del otoño de 2004, a través del estudio de la composición botánica, la abundancia de especies y la cobertura basal (Braun-Blanquet, 1979). A partir de esta información se identificaron tres sitios, caracterizados cada uno de ellos por 
poseer composición florística parcialmente homogénea y de modo que sus coberturas fueran relativamente elevadas respecto a otros sectores del lugar de estudio. Asimismo, se realizó un detallado levantamiento topográfico. Con los datos obtenidos mediante ambos relevamientos se construyeron dos mapas: de comunidades vegetales y de altimetría. Integrando la información consignada en los mapas, se determinaron diversos escenarios en cada sitio. En esta instancia se midió, en todos los escenarios, la masa aérea de la vegetación.
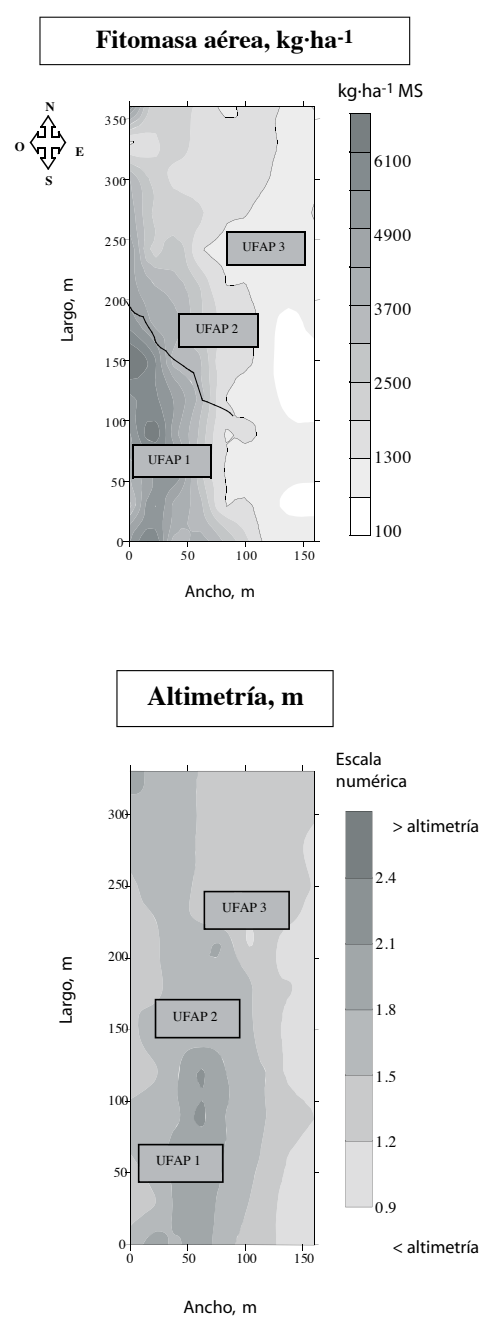

Figura 1. Ubicación de las unidades forrajeras de alta productividad (UFAP) en el área de estudio $\left(33^{\circ} 01^{\prime} \mathrm{S}\right.$ y $60^{\circ} 53^{\prime} \mathrm{O}$ ). Datos obtenidos en marzo de 2004.

Figure 1. Outline of the location of high productive foraging units (HPFU) $\left(33^{\circ} 01^{\prime} \mathrm{S}\right.$ and $\left.60^{\circ} 53^{\prime} \mathrm{W}\right)$ were obtained in March of 2004.
Cuadro 1. Características de las unidades forrajeras de alta productividad (UFAP), ubicadas en Santa Fe, Argentina $\left(33^{\circ} 01^{\prime} \mathrm{S}\right.$ y $\left.60^{\circ} 53^{\prime} \mathrm{O}\right)$.

Table 1. Characteristic of forrage units of high production (UFAP) located in Santa Fe, Argentina $\left(33^{\circ} 01^{\prime} \mathrm{S}\right.$ and $\left.60^{\circ} 53^{\prime} \mathrm{W}\right)$.

\begin{tabular}{lccc}
\hline Característica & \multicolumn{3}{c}{$\begin{array}{c}\text { Unidades forraje de alta } \\
\text { productividad }\end{array}$} \\
& UFAP 1 & UFAP 2 & UFAP 3 \\
Dimensión, m & $12 \times 18$ & $8 \times 12$ & $6 \times 18$ \\
Area, $\mathrm{m}^{2}$ & 216 & 96 & 108 \\
Pendiente, \% & 0,6 & 1,5 & 1,1 \\
\hline
\end{tabular}

Posteriormente se seleccionó un escenario de máxima productividad por sitio, respetando las siguientes condiciones: 1 . tamaño mayor a $1 \mathrm{~m}^{2}$ y 2. distribución espacial superior al $30 \%$ en el escenario. Los tres escenarios seleccionados se designaron como unidades forrajeras de alta productividad (UFAP). Las mismas presentaron cierta homogeneidad general en las siguientes variables de caracterización: productividad forrajera, abundancia de las especies clasificadas por grupo funcional y cobertura basal. La localización y delimitación de las UFAP se hizo a través de un georreferenciamiento, utilizando un navegador portátil (GPS) (Figura 1 y Cuadro 1).

Pese a la señalada homogeneidad general, el estudio integral de las variables utilizadas para caracterizar la vegetación permitió reconocer ciertas diferencias dentro de cada UFAP. Usando el índice de Braun-Blanquet, que intega abundancia y cobertura, se distinguió una o más unidades de combinación (BraunBlanquet, 1979). Correspondieron tres unidades de combinación a la UFAP 1, cuatro a la UFAP 2 y una a la UFAP 3.

Suelo

La diferente ubicación paisajística de las tres UFAP se correspondió con una diversidad de suelos. La UFAP 1 se encuentra sobre un suelo Argiudol vértico serie Roldán, la 2 sobre una fase peor drenada del anterior y la 3 sobre un Natracualf típico serie Zavalla.

A partir de los $40 \mathrm{~cm}$ de profundidad el suelo de la serie Roldán posee un horizonte B fuertemente textural. El B21t $(40-90 \mathrm{~cm})$ es 
la porción más enriquecida en arcilla de ese estrato (alrededor de un $50 \%$ ). Los valores de sodio son reducidos en todo el perfil del suelo y el pH es algo ácido en superficie y cercano a la neutralidad en los estratos más profundos.

El suelo de la serie Zavalla presenta un A2 en superficie $(0-20 \mathrm{~cm})$ con un contenido de limo muy elevado (76\%) y signos evidentes de limitaciones de drenaje (moteados, concreciones ferromanganésicas). Sigue a continuación un horizonte fuertemente textural $(55,6 \%$ de arcilla en el B21t). Todo el B tiene alto tenor de sodio (PSI superior al $15 \%$ ) y pH, alcalino, superior a 8 (Mosconi et al., 1981).

\section{Manejo de la pradera}

El área fue sometida a pastoreo rotativo con un rodeo de cría de vacas Hereford. Se pastoreó con carga promedio de $600 \mathrm{~kg} \cdot \mathrm{ha}^{-1}$, peso vivo, a partir de la acumulación de más de $1500 \mathrm{~kg} \cdot \mathrm{ha}^{-1}$ de materia seca, dejando un remanente de $6-7 \mathrm{~cm}$ $\left(800 \pm 150 \mathrm{~kg} \cdot \mathrm{ha}^{-1}\right.$ de materia seca). El tiempo de descanso varió estacionalmente según las tasas de crecimiento de las especies.

\section{Análisis y caracterización espacial}

Para establecer los puntos de muestreo de las UFAP, en cada una de ellas se trazó una retícula regular. De esta forma, se determinaron sobre el terreno una serie de puntos equidistantes entre sí a un metro, dando lugar a 216, 96 y 108 puntos muestrales para las UFAP 1 , 2 y 3 , respectivamente. Los puntos fueron referenciados sobre un origen de coordenadas previamente establecido en uno de los vértices de cada UFAP. En ellos se realizó un detallado $( \pm 1 \mathrm{~cm})$ relevamiento topográfico.

Se estimó la fitomasa aérea total por el método de capacitancia. Este método basa su funcionamiento en la diferencia de constante dieléctrica que existe entre el aire y la biomasa vegetal. Los cambios de capacitancia provocados por el reemplazo del aire por material vegetal debajo del cabezal medidor del instrumento, permitieron establecer una relación entre capacitancia y fitomasa aérea.

Con el fin de evaluar la importancia relativa de las especies dominantes dentro de cada UFAP, se determinó la abundancia y el grado de cobertura de las mismas, según el sistema de Braun-Blanquet (1979). Las mediciones se realizaron en $1 \mathrm{~m}^{2}$, al finalizar cada estación de crecimiento.

Para determinar la posible influencia de la napa freática en el drenaje se midió la profundidad de la misma. En un sector de cada UFAP se hizo un pozo cilíndrico de $2 \mathrm{~m}$ de profundidad y se introdujo un tubo de acrílico de $15 \mathrm{~cm}$ de diámetro que llegaba hasta la base del pozo, mientras que el extremo superior sobresalía encima de la superficie. Con una regla graduada introducida en el tubo se registró la distancia desde la superficie del suelo a la napa freática. Esta medición se realizó en seis y cinco oportunidades en otoño y en las demás estaciones del año, respectivamente. Las mediciones se realizaron con una separación entre dos registros consecutivos entre 11 y 22 días, de acuerdo a las condiciones de drenaje del área.

Para llevar a cabo los análisis geoestadísticos se empleó el programa SURFER (versión 5.01, 1994, Sandy, Utah, EUA). La base de datos disponibles fueron los valores de los puntos de coordenadas de la grilla asignada para cada UFAP, en relación a las siguientes mediciones: fitomasa aérea, clasificación florística y altimetría de precisión.

Las relaciones entre las características de la vegetación (unidades de combinación y fitomasa aérea) y la topografía de precisión se estudiaron matemáticamente por análisis de regresión lineal $(\mathrm{p}<0,05)$. Los análisis de correlación y regresión se realizaron con el programa estadístico INFOSTAT/ PROFESIONAL (Universidad Nacional de Córdoba, Argentina, 2001).

\section{Resultados}

Las temperaturas mensuales y las precipitaciones del área de trabajo se exponen en el Cuadro 2, tanto las del período de estudio como los promedios históricos durante 25 años, en igual distribución anual. Las precipitaciones ocurridas en dicho período alcanzaron un valor 
de $802 \mathrm{~mm}$, mientras que la media histórica fue de $987,5 \mathrm{~mm}$. El monto anual de lluvias resultó un $19 \%$ inferior a dicho promedio. La distribución temporal del desbalance fue irregular. El invierno y la primavera fueron las estaciones más deficitarias en relación a la media histórica $(57 \%$ y $40 \%$ de disminución, respectivamente). El análisis de los datos de las temperaturas medias mensuales muestra una variación de $3,14 \pm 0,47^{\circ} \mathrm{C}$ con respecto a los datos promedios anuales (Cuadro 2).

Las UFAP se hallaban situadas en diferentes posiciones relativas del paisaje. La UFAP 1 era parte de un sector más alto y mejor drenado que el que ocupaban las otras dos. La napa freática se mantuvo a una profundidad mayor a $1 \mathrm{~m}$ durante todo el año. La UFAP 2 se ubicó a $150 \mathrm{~m}$ de distancia de la primera; en una posición altimétrica entre 0,3 y $0,7 \mathrm{~m}$ más baja que UFAP 1 , con una napa freática que osciló, alrededor de la profundidad de $1 \mathrm{~m}$, a lo largo del año. La UFAP 3 correspondió a un área unos 0,6 m más baja que la UFAP 2, con grandes limitaciones de drenaje, suelos de $\mathrm{pH}$ alcalino, salinidad desde la superficie y napa freática a una profundidad inferior a $1 \mathrm{~m}$.

La microaltimetría de detalle muestra menor variabilidad topográfica en la UFAP 3 que en las otras dos unidades (Figura 2). Esto pone de manifiesto que los movimientos de los materiales en superficie ocurrieron a través de distancias reducidas, jerarquizando las relaciones dentro de los microrelieves.

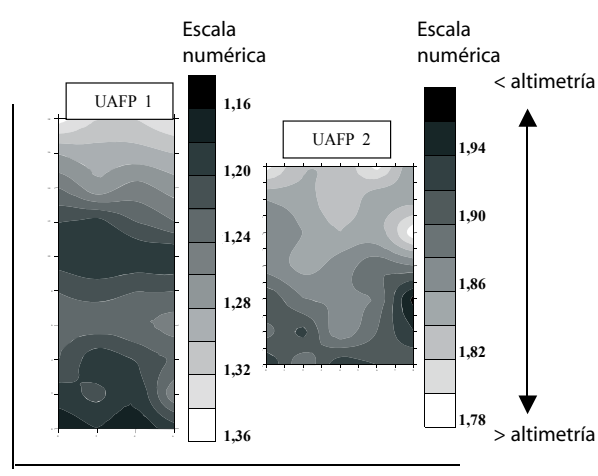

Ancho, $\mathrm{m}$

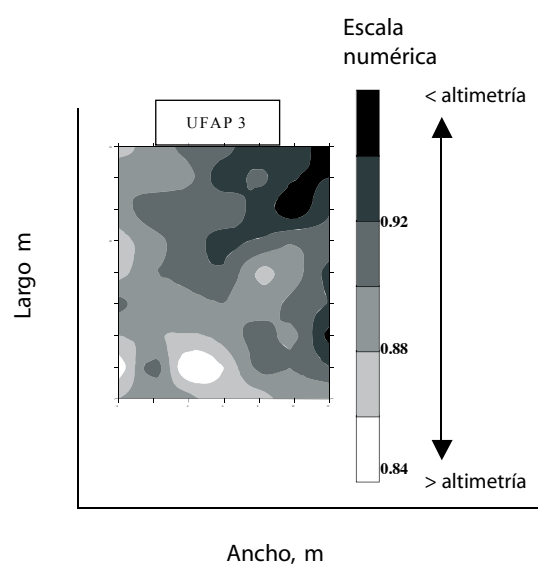

Figura 2. Microaltimetría de detalle en cada unidad forrajera de alta productividad (UFAP), sector ubicado en Santa Fe, Argentina a $33^{\circ} 01^{\prime} \mathrm{S}$ y a $60^{\circ} 53^{\prime} \mathrm{O}$. Datos obtenidos en marzo de 2004.

Figure 2. Microaltimetry of detail in each high productive foraging units (HPFU) located in Santa Fe Argentina at $33^{\circ} 01^{\prime} \mathrm{S}$ and $60^{\circ} 53^{\prime} \mathrm{W}$ were obtained in March of 2004.

Cuadro 2. Precipitaciones y temperaturas medias mensuales registradas en el año de estudio y promedios en el período

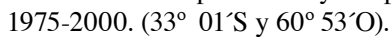

Table 2. Precipitation and annual distribution of mean temperature in the period of study and average for 25 years

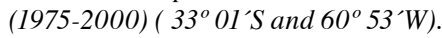

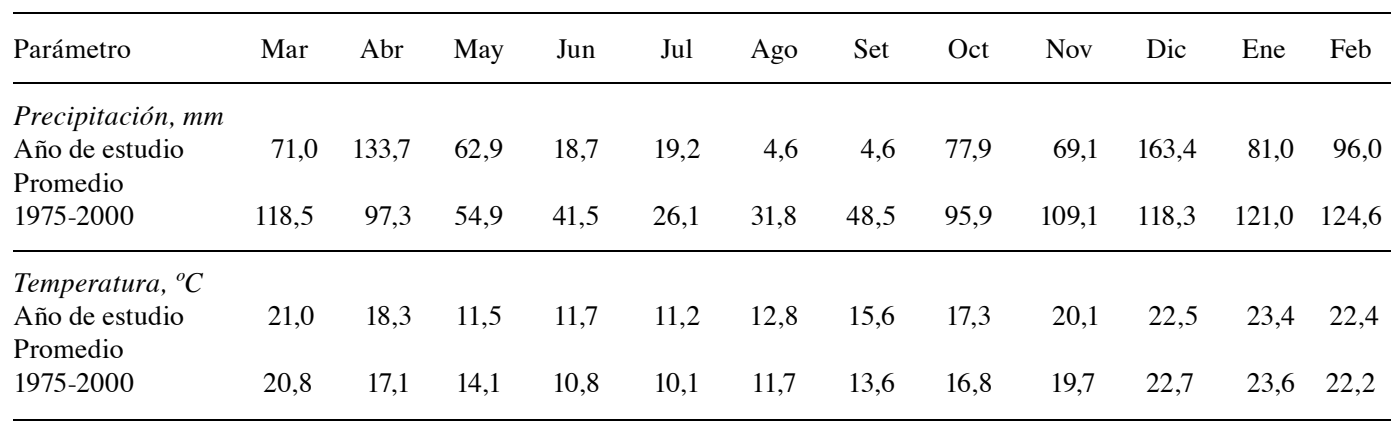


Cuadro 3. Principales especies presentes en las unidades forrajeras de alta productividad (UFAP) y sus valores de abundancia y cobertura. Datos obtenidos entre marzo y diciembre de 2004 . El sector ubicado a $33^{\circ} 01^{\prime} \mathrm{S}$ y a $60^{\circ} 53^{\prime} \mathrm{O}$.

Table 3. Abundancy-cover (Braun-Blanquet, 1979) of the main grassland species in high productive foraging units (HPFU). Measurements were done between March and December, 2004. The sector was located $33^{\circ} 01^{\prime} \mathrm{S}$ and to $60^{\circ} 53^{\prime} \mathrm{O}$.

\begin{tabular}{|c|c|c|c|c|c|c|c|c|c|c|c|c|c|c|c|c|}
\hline \multirow[b]{3}{*}{ Especies } & \multicolumn{16}{|c|}{ Unidades de combinación ${ }^{1}$} \\
\hline & \multicolumn{4}{|c|}{ Otoño } & \multicolumn{4}{|c|}{ Invierno } & \multicolumn{4}{|c|}{ Primavera } & \multicolumn{4}{|c|}{ Verano } \\
\hline & 1 & 2 & 3 & 4 & 1 & 2 & 3 & 4 & 1 & 2 & 3 & 4 & 1 & 2 & 3 & 4 \\
\hline & \multicolumn{16}{|c|}{$U F A P 1$} \\
\hline Paspalum dilatatum & 3 & 2 & 3 & & 3 & 2 & 3 & & 4 & 4 & 5 & & 4 & 4 & 5 & \\
\hline Setaria geniculata & 0 & 0 & 0 & & 0 & 0 & 0 & & 0 & 0 & 0 & & 2 & 3 & 1 & \\
\hline Bothiocloa laguroides & 0 & 0 & 0 & & 0 & 0 & 0 & & 0 & 1 & 0 & & 2 & 2 & 1 & \\
\hline Panicum milioides & 0 & 0 & 0 & & 0 & 0 & 0 & & 1 & 2 & 1 & & 2 & 3 & 1 & \\
\hline Stipa hyalina, S. neesiana & 2 & 3 & 4 & & 2 & 3 & 4 & & 2 & 3 & 2 & & 1 & 2 & 1 & \\
\hline Hordeum stenostachys & 5 & 4 & 4 & & 5 & 4 & 5 & & 5 & 3 & 2 & & 0 & 0 & 1 & \\
\hline Bromus catharticus & 5 & 1 & 5 & & 5 & 1 & 5 & & 3 & 2 & 5 & & 0 & 0 & 1 & \\
\hline Lolium multiflorum & 3 & 1 & 2 & & 3 & 1 & 2 & & 2 & 1 & 2 & & 0 & 0 & 0 & \\
\hline Graminoides (Carex, Cyperus, Juncus) & 1 & 1 & 1 & & 3 & 1 & 0 & & 3 & 0 & 1 & & 3 & 0 & 3 & \\
\hline \multirow[t]{2}{*}{ Cynodon dactylon } & 0 & 0 & 0 & & 0 & 0 & 0 & & 0 & 0 & 0 & & 1 & 0 & 0 & \\
\hline & \multicolumn{16}{|c|}{ UFAP 1} \\
\hline Paspalum dilatatum & 3 & 2 & 3 & 1 & 1 & 0 & 1 & 0 & 4 & 3 & 2 & 1 & 4 & 4 & 3 & 3 \\
\hline Setaria geniculata & 0 & 0 & 0 & 0 & 0 & 0 & 0 & 0 & 1 & 1 & 0 & 0 & 1 & 1 & 1 & 1 \\
\hline Panicum milioides & 0 & 0 & 0 & 0 & 0 & 0 & 0 & 0 & 0 & 3 & 3 & 1 & 0 & 3 & 3 & 1 \\
\hline Chloris halophila & 0 & 2 & 0 & 0 & 0 & 2 & 0 & 0 & 1 & 4 & 4 & 4 & 2 & 5 & 4 & 4 \\
\hline Stipa hyalina, S. neesiana & 0 & 3 & 2 & 1 & 0 & 2 & 1 & 1 & 4 & 3 & 1 & 1 & 3 & 2 & 1 & 1 \\
\hline Hordeum stenostachys & 5 & 4 & 4 & 2 & 5 & 4 & 4 & 3 & 4 & 3 & 3 & 2 & 0 & 0 & 0 & 0 \\
\hline Bromus catharticus & 5 & 2 & 4 & 1 & 5 & 3 & 5 & 2 & 3 & 2 & 3 & 1 & 0 & 0 & 0 & 0 \\
\hline Lolium multiflorum & 3 & 1 & 2 & 0 & 3 & 1 & 2 & 0 & 1 & 1 & 0 & 0 & 0 & 0 & 0 & 0 \\
\hline Graminoides (Carex, Cyperus, Juncus) & 4 & 3 & 0 & 3 & 4 & 3 & 0 & 3 & 3 & 0 & 0 & 0 & 3 & 3 & 0 & 0 \\
\hline \multirow[t]{2}{*}{ Cynodon dactylon } & 2 & 2 & 0 & 0 & 0 & 0 & 0 & 0 & 2 & 3 & 3 & 2 & 4 & 3 & 4 & 4 \\
\hline & \multicolumn{16}{|c|}{$U F A P 1$} \\
\hline Cynodon dactylon & 3 & & & & 2 & & & & 5 & & & & 5 & & & \\
\hline
\end{tabular}

${ }^{1}$ Unidades de combinación y números ( 0 a 5) según el sistema abundancia-cobertura de Braun-Blanquet (1979), donde $0=$ ausente, $1=$ muy escasa, $2=$ escasa, $3=$ no numerosa, $4=$ numerosa y $5=$ muy numerosa.

${ }^{1}$ Units of combination and numbers (0 to 5) according to system abundance-covering of Braun-Blanquet (1979), where $0=$ absentee, $1=$ very scarce, $2=$ scarce, $3=$ not numerous, $4=$ numerous and $5=$ very numerous.

Se encontraron 14 especies de plantas dominantes en todo el sector bajo estudio. En el Cuadro 3 se exponen los resultados de los censos estacionales de vegetación según UFAP. Dentro de cada una de éstas se discriminó de acuerdo con las unidades de combinación reconocidas.

Los mapas de distribución de la vegetación según la estación del año muestran que en las UFAP 1 y 2 las unidades de combinación variaron su disposición espacial en el transcurso del estudio (Figura 3).

Del total de especies relevadas en todo el sitio de trabajo, el $92 \%$ se presentó tanto en la UFAP 1 como en la UFAP 2. En ambas unidades forrajeras el $46 \%$ de las especies perteneció al grupo funcional gramíneas C4, el 31\% al grupo gramíneas $\mathrm{C} 3$ y el 23\% restante correspondió al grupo graminoides. Es importante destacar que Bothriocloa laguroides sólo estuvo presente en la UFAP 1 y que Chloris halophyla exclusivamente se registró en la UFAP 2. La especie Cynodon dactylon fue exclusiva en la UFAP 3 y definió la única unidad de combinación reconocida.

En las UFAP 1 y 2 la cantidad de fitomasa aérea alcanzó valores mínimos durante el invierno $\left(1.200 \pm 102,1.000 \pm 128 \mathrm{~kg} \cdot \mathrm{ha}^{-1}\right.$, en UFAP $1 \mathrm{y}$ 2 respectivamente) y máximos en la primavera $\left(2.800 \pm 850\right.$ y $2.600 \pm 800 \mathrm{~kg} \cdot \mathrm{ha}^{-1}$, en UFAP 1 y 2 respectivamente). En la UFAP 1 la distribución espacial de la fitomasa aérea tuvo la mayor homogeneidad en invierno; en la UFAP 
UAFP 1

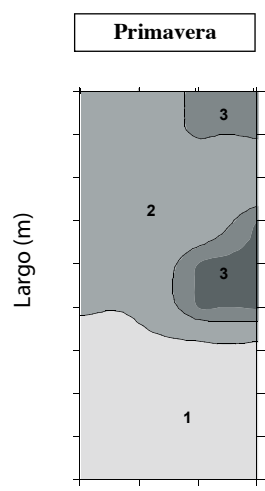

Ancho, m

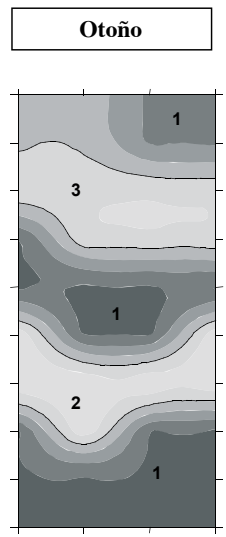

Ancho, m

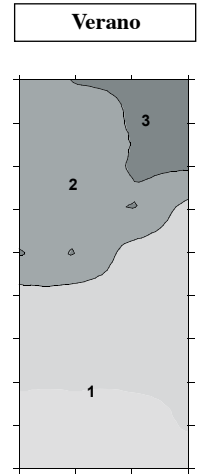

Ancho, m
UFAP 2
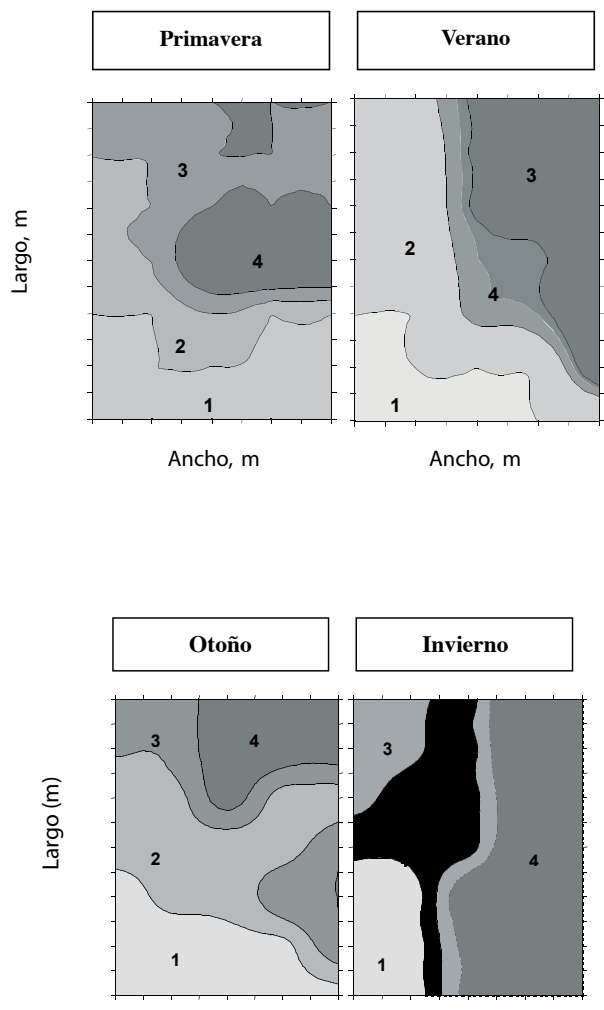

Ancho, m

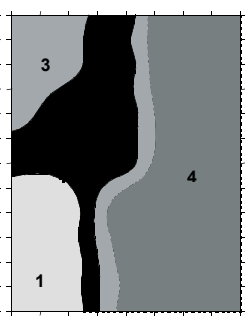

Ancho, m

Figura 3. Distribución espacial de las unidades de combinación en las unidades forrajeras de alta productividad (UFAP), según la estación del año. Los números representan las unidades de combinación, sus valores de abundancia y cobertura según Braun-Blanquet (1979). UFAP 1, sector más alto y mejor drenado, UFAP 2, sector ubicado en una posición altimétrica entre 0,3 y 0,7 m más baja que UFAP 1 .

Figure 3. Space distribution of combination units in species in high productive foraging units (HPFU) according to the season. Numbers indicate combination units and their abundance and cover values according to Braun-Blanquet (1979). HPFU 1 was the highest, well drained sector, and HPFU 2 was located between 0.3 and $0.7 \mathrm{~m}$ lower than HPFU.

2 esta situación se verificó en otoño e invierno. La producción forrajera estacional de la UFAP 3 $\left(1.680 \pm 287 \mathrm{~kg} \cdot \mathrm{ha}^{-1}\right.$ de materia seca, $2.345 \pm$ $358 \mathrm{~kg} \cdot \mathrm{ha}^{-1}$ de materia seca, en primavera $\mathrm{y}$ verano, respectivamente) estuvo condicionada por el comportamiento en el crecimiento de C. dactylon. Los valores medidos de fitomasa aérea en las UFAP tuvieron relaciones significativas con la posición altimétrica de los sitios evaluados (Cuadro 4).

Se observó que la altimetría se correlaciona significativamente con la composición específica de la comunidad vegetal y con la productividad forrajera. El coeficiente de correlación negativo entre las productividades y la altimetría sugiere que en algunos sitios de menor altura relativa de cada UFAP es probable encontrar mayores valores de fitomasa aérea.

\section{Discusión}

La existencia de heterogeneidad en la estructura de los pastizales se ha reconocido a diferentes 
Cuadro 4. Coeficientes de correlación de la microaltimetría con la producción de fitomasa aérea y de la microaltimetría con la distribución espacial de las unidades de combinación, en cada unidad forrajera de alta productividad (UFAP).

Table 4. Correlation coefficients obtained between microaltimetr and forage production and between microaltimetry and space distribution of combination units, in each high productive foraging units (HPFU), according to the season.

\begin{tabular}{|c|c|c|c|c|c|c|}
\hline \multirow[b]{3}{*}{ Parámetro } & \multicolumn{6}{|c|}{ Unidades forrajeras de alta productividad $^{1}$} \\
\hline & \multicolumn{2}{|c|}{ UFAP 1} & \multicolumn{2}{|c|}{ UFAP 2} & \multicolumn{2}{|c|}{ UFAP 3} \\
\hline & $\mathrm{rp}$ & $\mathrm{rp}$ & $\mathrm{rp}$ & & & \\
\hline Altimetría & 1 & 1 & 1 & & & \\
\hline Fitomasa aérea en el otoño & -0.20 & 0,22 & $-0,30 *$ & $0,08 *$ & - & - \\
\hline UC en el otoño & $-0,30 *$ & $0,06 *$ & $0,40 *$ & $0,02 *$ & - & \\
\hline Fitomasa aérea en el invierno & $-0,20$ & 0,23 & $-0,44 *$ & $0,01 *$ & - & - \\
\hline UC en el invierno & $0,64 *$ & $8,9 \times 10^{-6 *}$ & $0,38 *$ & $0,02 *$ & - & - \\
\hline Fitomasa aérea en la primavera & $-0,25$ & 0,18 & $-0,38 *$ & $0,03 *$ & $0,31 *$ & $0,01 *$ \\
\hline UC en la primavera & $0,45 *$ & $3,6 \times 10^{-3} *$ & $0,51 *$ & $1,9 \times 10^{-3} *$ & - & - \\
\hline Fitomasa aérea en el verano & $-0,37 *$ & $0,02 *$ & $-0,53 *$ & $1,2 \times 10^{-3} *$ & $0,45 *$ & $2,2 \times 10^{-*}$ \\
\hline UC en el verano & $0,58 *$ & $8,3 \times 10^{-5} *$ & 0,22 & 0,19 & - & - \\
\hline
\end{tabular}

'Unidades Forrajeras de Alta Productividad (UFAP): UFAP 1, sector alto, bien drenado; UFAP 2, sector en una posición altimétrica entre 0,3 y $0,7 \mathrm{~m}$ que UFAP 1 y UFAP 3 , sector en una posición altimétrica de $0,6 \mathrm{~m}$ más baja que UFAP 2. Datos obtenidos entre marzo y diciembre de 2004. * Coeficientes de correlación estadísticamente significativos $(\mathrm{p}<0.05)$.

${ }^{\prime}$ High Productive Foraging Units (HPFU): HPFU 1, high sector, well drainage; HPFU 2, sector in position altimetry between 0.3 y $0.7 \mathrm{~m}$ that HPFU 1 and HPFU 3, sector in position altimetry of $0.6 \mathrm{~m}$ lower that HPFU 2. Data were obtained between March and December of 2004. * Coefficient of correlation significant at $p<0.05$.

escalas (Pucheta et al., 2004). Cabido et al. (1997), Nai-Bregaglio et al. (2002) y Cingolani et al. (2003) determinaron que a nivel de lotes la estructura del pastizal está controlada por la intensidad del uso ganadero y por las características edáficas y topográficas. En el presente trabajo el pastizal fue sometido a una moderada intensidad de pastoreo, cuando logró acumular más de $1500 \mathrm{~kg} \cdot \mathrm{ha}^{-1}$ de materia seca, dejando un remanente de $800 \pm 150 \mathrm{~kg} \cdot \mathrm{ha}^{-1}$ de materia seca. Tal manejo permitió realizar pastoreos controlados con alta carga y mantener la composición botánica (Luisoni, 2003).

La heterogeneidad observada en las UFAP 1 y 2 se explica en parte por la vinculación de las características estudiadas de la vegetación con la posición altimétrica (Figura 3). Esto podría estar relacionado con el drenaje debido a que los sectores más bajos presentan mayores limitaciones para la normal evacuación de los excedentes hídricos del suelo. Precisamente, las condiciones ambientales de la parte más deprimida de la superficie bajo estudio (UFAP 3) dieron lugar a la adaptación de $C$. dactylon como una única especie vegetal.

Tongway et al. (2004) han aludido a un nuevo paradigma de circulación tridimensional de los flujos hídricos y a la formación de islas o parches de fertilidad, lo que supone un acrecentamiento de la heterogeneidad espacial del suelo y que la vegetación se reorganiza según esa heterogeneidad. Por otra parte, diversos autores enunciaron que pequeñas diferencias en la posición topográfica pueden generar cambios importantes en la dinámica del agua (León et al., 1979; Aguiar y Sala, 1999).

Puigdefábregas et al. (1999) y Gibon (2005) encontraron que el gradiente de pendiente es uno de los parámetros topográficos que correlaciona significativamente con las propiedades del suelo. Así, al crecer la concavidad, también lo hace el espesor del horizonte A. En cada UFAP se registraron valores promedios y desvíos similares a los señalados por estos autores $(27 \pm 3,4 \mathrm{~cm} ; 24 \pm 2,1 \mathrm{~cm}$ y $20 \pm 2,0 \mathrm{~cm}$, de profundidad del horizonte A para la UFAP 1, 2 y 3 respectivamente).

Sharma et al. (1998) encontraron que los sectores cóncavos poseían una mayor percolación del agua, escurrimiento despreciable y acumulación de aguas provenientes de áreas adyacentes relativamente más altas, siendo así mayor la precipitación efectiva. Del análisis de las unidades de combinación de especies en las UFAP 1 y 2 se observó que la cobertura de las gramíneas festucoideas (tipo C3) aumentó en 
los sectores altimétricos más bajos, en especial aquellas de comportamiento anual. Por otra parte, en los sitios más altos (diferencias altimétricas de $20 \mathrm{~cm}$ en la UFAP 1 y de 16 $\mathrm{cm}$ en la UFAP 2, entre el sector más elevado y el más cóncavo), predominó la cobertura de las gramíneas perennes no festucoideas (tipo C4) (Figura 3 a y b). Estos resultados se relacionan a lo documentado por Harper et al. (1965) quienes señalan la influencia diferencial de las variaciones en la microtopografía de la superficie del suelo sobre el éxito del establecimiento y crecimiento de diferentes especies.

La dinámica del agua del suelo, relacionada con la altimetría, son considerados dentro de los factores clave que influyen en la composición de especies de una comunidad vegetal, como lo manifiestan Sollins (1998) y Laurance et al. (1999) para otros ambientes. En este lugar, caracterizado por ser un sector deprimido, se observó que en los períodos de precipitaciones menores a las medias regionales (Cuadro 2), la menor altimetría en cada UFAP condicionó significativamente el crecimiento de las especies de plantas en cada unidad de combinación (Cuadro 4). La ubicación de la UFAP 2, intermedia en el sector, logró mayores ventajas ambientales respecto a la UFAP 1 , en los referidos períodos.

Desde la perspectiva del uso del recurso en estos ambientes, es importante conocer el funcionamiento productivo de la comunidad, condicionado por las unidades de vegetación participantes en la comunidad (D’Angelo, 2002). Para Forman y Gordon (1986), los cambios en la proporción de estas unidades podrían generar estados productivamente más deseables del pastizal.

Finalmente, los resultados obtenidos sugieren que las condiciones microtopográficas, en ambientes planos, son ecológicamente significativas. Esto debido a que están altamente correlacionados con la distribución de las especies vegetales y con la productividad del recurso forrajero. Oliveira-Filho et al. (1998) y Johnstone (1992), arribaron a similares conclusiones, desarrollando sus estudios en ambientes contrastantes al de este trabajo.
Por lo tanto, se considera que la heterogeneidad fisonómica de los pastizales de áreas deprimidas está altamente relacionada con las variaciones microtopográficas. La complejidad de las relaciones entre los parámetros topográficos y los de la vegetación hace necesaria la evaluación en detalle de diversas variables para caracterizar correctamente a cada sector.

\section{Resumen}

A menudo, las comunidades vegetales presentes en los paisajes deprimidos de la Pampa Ondulada son espacialmente heterogéneas. El objetivo de este trabajo fue establecer si existe relación entre las características estructurales y funcionales de la vegetación con la topografía, en un área mal drenada. El estudio se realizó en un sector próximo a una cañada perteneciente a la cuenca del arroyo Ludueña (Zavalla, Santa Fe, Argentina). El recorrido del arroyo Ludueña se ubica entre los $32^{\circ} 46^{\circ}$ y $33^{\circ} 07^{\prime}$ de latitud sur y $60^{\circ} 39^{\prime}$ y $61^{\circ} 07^{\prime}$ de longitud oeste. La identificación de las comunidades vegetales y un relevamiento topográfico permitieron reconocer tres unidades forrajeras de alta productividad (UFAP). En cada una se midió la productividad primaria neta aérea, la abundancia y el grado de cobertura, y se establecieron diversas unidades de vegetación. Los resultados sugieren que en los ambientes deprimidos la cobertura y la abundancia de la vegetación están altamente relacionadas con la microtopografía.

Palabras clave: Ambientes deprimidos, comunidades vegetales, topografía, unidades forrajeras.

\section{Literatura citada}

Aguiar, M.R., and O.E. Sala. 1999. Patch structure, dynamics and implications for the functioning of arid ecosystems. Trends in Ecology and Evolution 14:273-277.

Allen R.B., and P.H. Mc Intosh. 1997. The distribution of plants in relation to $\mathrm{pH}$ and salinity on inland saline/alkaline soils in Central Otago, New Zealand. New Zealand Journal of Botany 35:517-523.

Archer, S., D.S. Schimel, and E.H. Holland. 1995. Mechanisms of shrubland expansion: Land use, climate, or $\mathrm{CO}_{2}$. Climatic Change 29:91-99. 
Batista, W.B. y R.J.C. León. 1992. Asociación entre unidades vegetales y algunas propiedades del suelo en el centro de la depresión del salado. Ecología Austral 2:47-55.

Behnke Jr., R.H., I. Scoones, and C. Kerven (eds.). 2000. Range Ecology at Disequilibrium. Overseas Development Institute and Others Institutions. London, UK. 167 pp.

Braun-Blanquet, J. 1979. Fitosociología-Bases para el Estudio de las Comunidades Vegetales. Blume, Madrid, España. 420 pp.

Cabido, M., N. Ateca, M.E. Ategiano, and A.M. Anton. 1997. Distribution of C3 and C4 grasses along an altitudinal gradient in central Argentina. Journal of Biogeography 24:197204.

Chaneton, E.J., and R.S. Lavado. 1996. Soil nutrients and salinity after long-term grazing exclusion in a Flooding Pampa grassland. Journal of Range Management 49:182-187.

Cingolani, A.M., M. Cabido, D. Renison, and V. SolísNeffa. 2003. Combined effects of environment and grazing on vegetation structure in Argentine granite grasslands. Journal Vegetation Science 14:223-232.

Coffin, D., and W. Lavenroth. 1988. The effects of disturbance size and frequency on a short grass plant community. Ecology 69:1609-1617.

Corwin, D.L., S.R. Kaffka, J.D. Oster, O. Hopmans, Y. Mori, J.W. Van Groenigen, C. Van Kessel, and S.M. Lesch. 2003. Assessment and fieldscale mapping of soil quality properties of a saline-sodic soil. Geoderma 14:231-259.

D’Angelo, C. 2002. Principios generales de la ordenación predial. p. 897-921. En. J. Gastó, P. Rodrigo e I. Aránguiz (eds.). Ordenación Territorial, Desarrollo de Predios y Comunas Rurales. Facultad de Agronomía e Ingeniería Forestal, Pontificia Universidad Católica de Chile. LOM Ediciones. Santiago, Chile.

Deregibus, V.A., E. Jacobo, and O.E. Ansín. 2001. Grassland use and plant diversity in grazed ecosystems. p. 879-882. In. Proceeding of the XIX International Grassland Congress, 2001 Brazil.

Forman, R., and M. Gordon. 1986. Landscape Ecology. John Wiley and Sons, New York, USA. 618 pp.

Gibon, A. 2005. Managing grassland for production, the environment and the landscape. Challenges at the farm and the landscape level. Livestock Production Science 96:11-31.

González-Bernaldez, F. 1981. Ecología del Paisaje. Blume, Madrid, España. 223 pp.

Goslee, S.C., R.P. Brooks, and C.A. Cole. 1997. Plants as indicators of wetland water source. Plant Ecology 131:199-206.
Harper, J.L., J.T. Williams, and G.R. Sagar. 1965. The behaviour of seed in soil. Part 1: The heterogeneity of soil surfaces and its role in determining the establishment of plant from seed. Journal of Ecology 53:273-286.

Hartnett, D., and P.A. Fay. 1998. Plant population patterns and processes. p. 81-98. In. A.K Knapp, J.M. Briggs, D.C. Hartnett, and S.L. Collins (eds.). Grassland Dynamics. Oxford University Press. New York, USA. 386 pp.

INTA. 1983. Carta de suelos de la República Argentina; hoja 3360-20 y 21, Arroyo Seco y San Nicolás de los Arroyos. Instituto Nacional de Tecnología Agropecuaria, INTA, Buenos Aires, Argentina.

Johnstone, M.H. 1992. Soil-vegetation relationships in a Tabonuco forest community in the Luquillo Mountains of Puerto Rico. Journal Tropical Ecology 5:253-263.

Laurance, W.F., P.M. Fearnside, S.G. Laurance, P. Delamonica, T.E. Lovejoy, J.M. Rankinde Merona, J.Q. Chambers, and C. Gascon. 1999. Relationships between soils and Amazon forest biomass: a landscape-scale study. Forest Ecology Management 118:127-138.

León, R.J.C., S. Burkart y C. Movia. 1979. Relevamiento fitosociológico del pastizal del norte de la Depresión del Salado. Instituto Nacional de Tecnología Agropecuaria, INTA. Serie Fitogeografica $n^{\circ} 17$, pp. 11-93. Buenos Aires, Argentina.

Luisoni, L.H. 2003. Parque Chaqueño Oriental: Cambios en la vegetación provocados por el pastoreo. Conferencia $2^{\circ}$ Congreso Nacional sobre Manejo de Pastizales Naturales. vol. 1, Resúmenes. San Cristóbal, Santa Fe, Argentina. $145 \mathrm{pp}$.

Milchunas, D.G., and W.K. Lauenroth. 1989. Threedimensional distribution of plant biomass in relation to grazing and topography in the short grass steppe. Oikos 55:82-86.

Mosconi, F., J. Priano, N. Hein, G. Moscatelli, J. Salazar, T. Gutiérrez y L. Cáceres. 1981. Mapa de suelos de la Provincia de Santa Fe. Tomo I Ed: INTA-MAG, Santa Fe, Argentina. 248 pp.

Nai-Bregaglio, M., E. Pucheta y M. Cabido. 2002. El efecto del pastoreo sobre la diversidad florística y estructural en pastizales de montaña del centro de Argentina. Revista Chilena de Historia Natural 75:613-623.

Oliveira-Filho, A., N. Curi, E.A. Vilela, and D. Carvalho. 1998. Effects of canopy gaps, topography and soils on the distribution of woody species in a central Brazilian deciduous dry forest. Biotropica 56:362-375.

Onkware, A.O. 2000. Effect of soil salinity on plant distribution and production at Laburu delta, 
Lake Bogoria National Reserve, Kenya. Austral Ecology 25:140-149.

Pastor, J, M.A. Stillwell, and D. Tilman. 1987. Little bluestem litter dynamics in Minnesota old field. Oecologia 72:327-330.

Pastor, J., and W.M. Post. 1993. Linear regressions do not predict the transient responses of eastern North American forests to $\mathrm{CO}_{2}$-induced climate changes. Climate Change 23:111-119.

Pucheta, E., F. Ferrero, L. Heil y C. Schneider. 2004. Modelos de regresión para la estimación de la biomasa aérea en un pastizal de montaña de Pampa de Achala (Córdoba, Argentina). Agriscientia 21(1):23-30.

Puigdefábregas, J., A. Solé-Benet, L. Gutiérrez, G. Del Barrio, and M. Boer. 1999. Scales and processes of water and sediment redistribution in drylands: results from the Rambla Honda field site in Southeast Spain. Earth-Science Reviews 48:39-70.

Sala, O.E., M. Oesterheld, R.J.C. León, and A.
Soriano. 1986. Grazing effects upon plant community structure in subhumid grasslands of Argentina. Vegetatio 67:27-32.

Sánchez-Sánchez, O., and G. Islebe. 2002. Tropical forest communities in southeastern. Mexico. Plant Ecology 158:183-200.

Sharma, B.D., S. Mukhopadhyay, and P. Sidhu. 1998. Microtopographic controls on soil formation in the Punjab region, India. Geoderma 81:357-368.

Sollins, P. 1998. Factors influencing species composition in tropical lowland rain forest:does soil matter. Ecology 79:23-30.

Stephenson, N.L. 1990. Climatic control of vegetation distribution: The role of the water balance. Nature 135:649-670.

Tongway, D.J., J. Cortina y F.T. Maestre. 2004. Heterogeneidad espacial y gestión de medios semiáridos. Ecosistemas 2004/1 www. revistaecosistemas.net/articulo.asp? Id=88\&Id_ categoría $=1 \&$ tipo=portada. (Consultado en diciembre de 2006). 